\title{
The Immune Response to Papillomavirus During Infection Persistence and Regression
}

\author{
Merilyn H. Hibma*
}

Department of Microbiology and Immunology, University of Otago, P.O. Box 56, Dunedin, New Zealand

\begin{abstract}
Human papillomavirus (HPV) infections cause a significant global health burden, predominantly due to HPVassociated cancers. HPV infects only the epidermal cells of cutaneous and mucosal skin, without penetration into the dermal tissues. Infections may persist for months or years, contributed by an array of viral immune evasion mechanisms. However in the majority of cases immunity-based regression of HPV lesions does eventually occur. The role of the innate immune response to HPV in persistence and regression of HPV infection is not well understood. Although an initial inflammatory infiltrate may contribute to disease regression, sustained inflammation at the HPV-induced lesions, characterized by macrophage and neutrophil infiltration, has been observed in persistence. Pathogen-associated molecular patterns (PAMPs) are important in innate recognition. The double stranded DNA and an L1 and L2 capsid components of the HPV virion are potential PAMPs that can trigger signaling through cellular pattern recognition receptors, including toll-like receptors (TLR). TLR expression is increased in regressing HPV disease but is reduced in persistent lesions, suggesting a role for TLR in HPV regression. With regard to the adaptive immune response, a key indicator of regression in humans is infiltration of the lesion with both CD4 and CD8 T cells. In individuals with persistent lesions, CD8 T cell and immune suppressive regulatory $\mathrm{T}$ cells (Tregs) infiltrate the infection site. There is no association between persistence or regression and the presence of serum antibodies to the viral capsid antigens of HPV. There is still much to be learned about the immunological events that trigger regression of HPV disease. Understanding the viral and host factors that influence persistence and regression is important for the development of better immunotherapeutic treatments for HPVassociated disease.
\end{abstract}

Keywords: Adaptive immunity, epidermis, immune evasion, innate immunity, papillomavirus, persistence, regression.

\section{REVIEW}

\section{Regression of HPV Lesions Frequently Occurs Months or Years After the Initial Infection}

Human papillomaviruses (HPV) cause a range of flat or proliferative lesions of the epithelium, having a type-specific tropism for cutaneous or mucosal epidermis. The natural history of HPV infection gives some insight into the interplay between the host and the virus. In contrast with an acute viral infection such as influenza, which is short-lived, highly productive and induces a strong immune response with anti-viral immunity, HPV infections are persistent and are poorly productive. Persistence of HPV is contributed to by a multitude of mechanisms to avoid detection by the host immune system and is indicative of immunological ignorance, inappropriate immunity or anergy.

Transient HPV infections are cleared within months following infection, however some infections may persist for years [1]. While for some HPV types there is infectionassociated pathology, primarily evidenced by cellular proliferation, this is not the case for all HPV infections. Indeed there is increasing evidence for a commensal status for at least some HPV types, in particular for members of the

*Address correspondence to this author at the Department of Microbiology and Immunology, University of Otago, P O Box 56, Dunedin, New Zealand; Tel: +64 3479 7726; Fax: +64 3479 7744;

E-mail: Merilyn.hibma@otago.ac.nz $\beta$ genus. There is also evidence that latency, a state of limited viral antigen expression and an absence of productive viral infection, can occur. HPV DNA can be detected in cells in the absence of any pathology, for instance in respiratory infection with HPV11 [2]. Furthermore, viral DNA can be detected following clearance of rabbit oral papillomavirus lesions [3].

The immune system has comparatively little exposure to late viral antigens. Depending on the HPV type and its tissue tropism, virion production can be limited. Although some cutaneous HPV $\alpha$ types are highly productive, $\beta$ type infections are less so. Mucosal HPV infections, especially laryngeal papillomas, generally produce few virions. Furthermore, HPV virions are shed with dead skin cells rather than released, as is the case for lytic viruses. Consequently, the quantity of late antigens presented to the immune system also may be limited.

The Epithelium is Rich in Immune Cells that can be Activated in Response to Viral Infection

The squamous epithelium is subdivided into two distinct regions, the epidermis and the dermis, separated by the epidermal basement membrane, and is populated with an array of immune cells (Fig. 1). The keratinocytes (KCs), which make up the majority of the cells in squamous epidermis, while not classical immune cells, do have some immune function. They can act as non-professional antigen presenting cells (APC), being competent to present peptides 


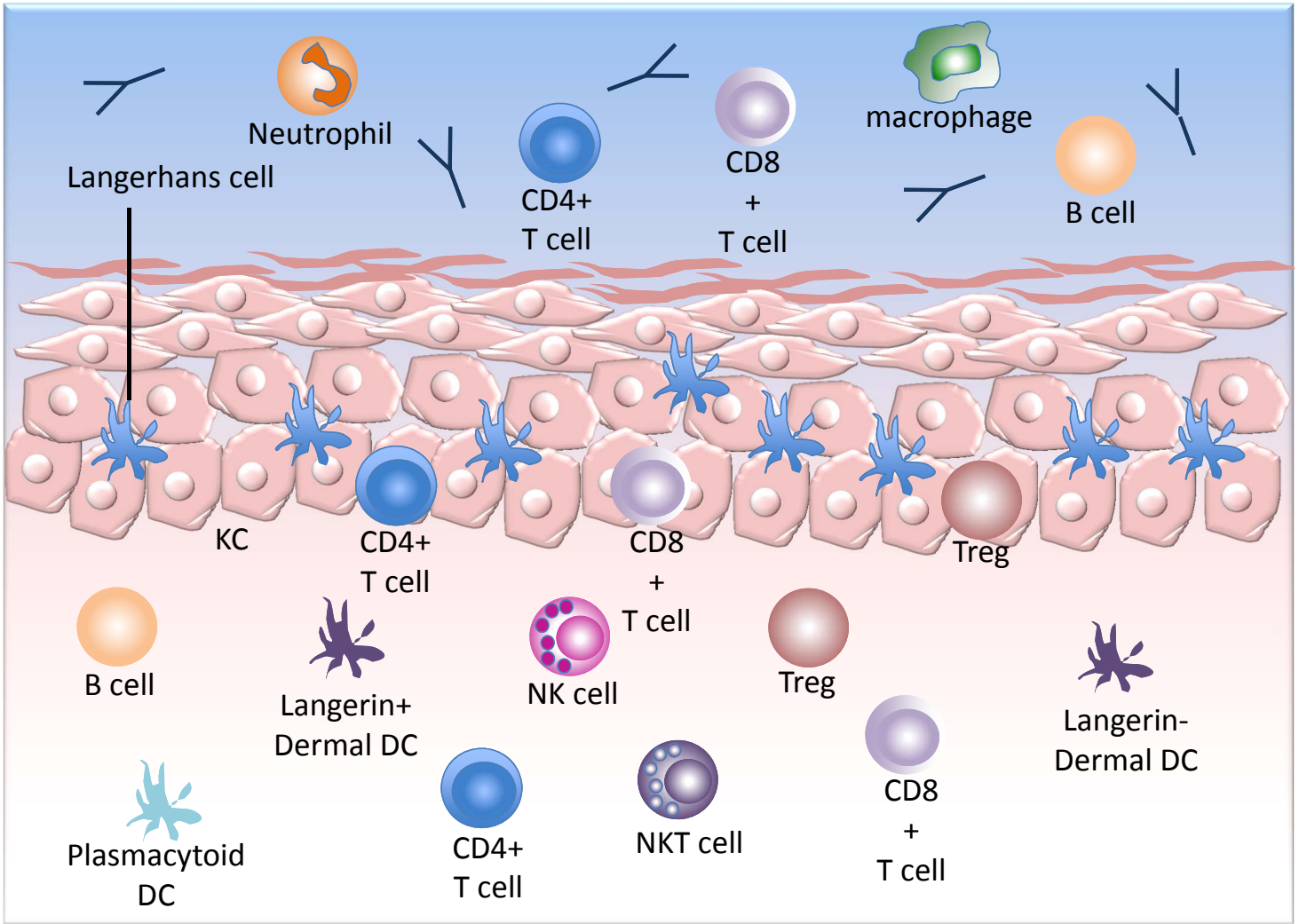

Fig. (1). The cells of the skin immune system. The epidermis is made up of keratinocytes with the capacity for some immune function, including cytokine secretion and the potential to act as 'non-professional' antigen presenting cells. The professional antigen presenting Langerhans cells also populate the epidermis, as do lymphocytes. Migrating Langerhans cells can be found in the dermis, along with dermal dendritic cells. Innate cells, such as NK and NKT cells and lymphocytes, populate normal dermis, whereas plasmacytoid dendritic cells are only present in pathological conditions including inflammation. The genital mucosa contains granulocytes, including neutrophils, and macrophages and lymphocytes.

in association with MHC class I and when expressed, MHC class II. KCs are also capable of secreting pro-inflammatory cytokines and chemokines and can induce the activation of CD4 and CD8+ memory $\mathrm{T}$ cells into a functional cytokinesecreting and cytotoxic state [4].

Although the majority of 'classical' immune cells are confined to the dermis in normal skin, some populations do reside in the epidermis. The predominant immune population in the epidermis is the Langerhans cells (LC), which are CD207+ professional APCs. T cells also can be found in the basal and suprabasal layers of the epidermis and are predominantly $\alpha / \beta$ TCR + memory T cells in the human [5].

Several subpopulations of APCs have been identified in the dermis. In addition to the langerin + LC migrating though the dermis, langerin + dermal dendritic cells (dDC) have been identified [6]. In the human, dermal migratory LC are distinguished from langerin+ $\mathrm{dDC}$ by high $\mathrm{CD} 1 \mathrm{a}$ and intermediate CD11c expression, whereas langerin+ $\mathrm{dDC}$ have intermediate CD1a and high expression of CD11c [7]. In the mouse, EpCAM expression on migratory LC distinguishes them from the langerin+ dDC [8]. The langerin $+\mathrm{dDC}$ can be further subdivided based on CD103 expression. The CD103+ population is considered essential for the presentation of antigen and initiation of an adaptive immune response in herpes simplex virus (HSV) infection in the mouse, and is capable of cross-presentation of exogenous antigen to $\mathrm{CD} 8+\mathrm{T}$ cells [9]. In accordance with those observations, the majority of langerin+ cells in the skin draining lymph nodes of humans have a phenotype consistent with them being $\mathrm{dDC}$ [7]. Other DC populations found in the skin have specialized functions, including plasmacytoid DC of the dermis, which secrete type I IFNs and inflammatory DC of inflamed epidermis, which secrete inflammatory mediators.

At the cervix, the stromal (dermal) lymphocytes include NKT, NK and B cells but are predominantly T cells, which are mostly beneath the basement membrane, at the epidermal-stromal junction [10]. The 'skin-resident' $\mathrm{T}$ cell population is significant (more than twice as many $\mathrm{T}$ cells than in the blood) and is proposed to have an important role in skin immune function $[11,12]$. Clonal expansion of skin resident $\mathrm{T}$ cells occurs as an immediate response to reinfection. During HSV infection, for example, antigen presentation to memory CD8 $+\mathrm{T}$ cells in the presence of $\mathrm{CD} 4+\mathrm{T}$ cells in the skin causes their proliferation, bypassing the involvement of lymphatic organs [13]. Following vaccinia virus infection, long-lived, non-recirculating CD8+ skin-resident memory cells can be found throughout the skin, which offer rapid protection against re-infection, even in the absence of CD4+ T cells [14]. The memory $\mathrm{T}$ cells in the skin provide an immediate response to re-exposure to a viral pathogen. Immune activation in the lymph node that is 
normally required for $\mathrm{T}$ cell priming is not necessary for clonal expansion of memory cells in the skin [15].

In addition to the cells resident in the epithelium, the genital tract is mucosal and the mucus layer that covers the epidermis is rich in innate and adaptive immune effectors. The immune cells in normal vaginal lavages consist of predominantly of granulocytes including neutrophils, with macrophages and $\mathrm{T}$ and $\mathrm{B}$ cells also present [16]. Secretory antibodies are the major effector component of the adaptive immune response. Innate molecules such as defensins and cytokines are also present in the mucosa.

The Innate Immune System is the First Line of Recognition and Defense Following HPV Infection

The skin provides a barrier between the environment and other tissues of the body. A critical role for the skin immune system is to distinguish between organisms that pose a threat and those that mutually co-exist with the host. The skin is an immunologically active site, triggered by tissue damage, inflammation and immune recognition of pathogenassociated molecular patterns (PAMPs). The key characteristics of the immune response to HPV in persistent and in regressing lesions are summarized in Table $\mathbf{1 .}$

Infiltration of inflammatory cells occurs in response to tissue damage: HPV infection is associated with tissue damage. The minimum involvement is up to the basal layer of the epidermis, so that the virions can infect these dividing cells [17]. The tissue damage associated with skin wounding has an associated infiltration of inflammatory cells - initially neutrophils, then macrophages followed by $\mathrm{T}$ cells [18]. Keratinocytes, when stimulated, can release proinflammatory mediators to recruit cells and activate them to release cytokines. The role of inflammation in initial HPV infection is not well understood however there is some evidence that inflammatory factors and cells influence regression of disease or persistence. In rabbit papillomavirus infection TNF $\alpha$ expression, produced by infiltrating mononuclear cells, correlates with regression [19]. In contrast, in low-grade squamous intraepithelial lesions (LSIL) [20] and high-grade cervical neoplasia [21] inflammation, as characterized by increased numbers of macrophages in the lesion, is associated with persistence or progression. In addition, the location of the inflammatory cells appears to be important. Kovacic, et al. found that women positive for carcinogenic HPVs had greater inflammation (increased neutrophils and lymphocytes) in the superficial epithelium compared with the stroma, which contrasted with samples from uninfected skin and skin infected with noncarcinogenic types [22]. There are numerous variables that confound the understanding of the role of inflammation in HPV persistence or regression, including whether the inflammation is acute or chronic, the location of the inflammatory cells in the epidermis, the genotype of the infecting virus and the length of time that the virus has persisted in the host.

The immune system has evolved to recognize danger signals: Initial signaling of infection is mediated by immune recognition through PAMPs, which signal danger to the immune response. Many bacterial and viral components are PAMPs and initiate signaling through pattern recognition receptors (PRR) on APCs, assisting the immune system in the decision to initiate an immune response. Potential HPV PAMPs are the L1 and L2 protein-containing capsid and the $8 \mathrm{~kb}$ double-stranded circular DNA genome.

Viral PRR are specialized into cellular compartments and into recognition of different viral components. Cell surface toll-like receptors (TLRs) detect hydrophobic lipids and proteins, the endosome-located TLRs detect nucleic acids and the cytoplasmic RIG-1-like receptors (RLRs), the nucleotide oligomerisation domain-like receptors (NLRs) and DNA sensing AIM2 family members detect nucleotides. The dsDNA genome of HPV is a likely activator of endosome and cytoplasmic DNA sensors and CpG motifs in the genome that promote signaling through TLR9 have been also identified [23]. TLRs, RLRs and DNA sensing molecules regulate interferon and cytokine expression whereas NLRs and AIM2 function through the inflammasome to activate caspase-1, thereby inducing maturation of IL-1 $\beta$ and IL-18.

Human KCs constitutively express TLR1- 6 and 9 and 10 [24] but not TRL7 and 8. KCs also constitutively express pro-IL1 $\beta$, which can be converted to active IL-1 $\beta$ and secreted [25]. There is evidence of a role for TLR signaling in clearance of HPV infection. Expression of mRNA for

Table 1. Key Characteristics of the Immune Cells in Persistent Papillomavirus Infection and Regressing Disease

\begin{tabular}{|c|c|}
\hline Regression & Persistence \\
\hline $\begin{array}{l}\text { - } \quad \text { increased TLR expression } \\
\text { - } \mathrm{TNF} \alpha \text { production by infiltrating mononuclear cells } \\
\text { - } \quad \text { infiltration of antigen presenting cells } \\
\text { - } \mathrm{Th} 1 \text { response } \\
\text { - } \mathrm{CD} 4 \text { infiltrate } \\
\text { - } \mathrm{CD} 8 \text { infiltrate } \\
\text { - } \quad \text { high CD4:CD8 ratio } \\
\text { - } \quad \text { cell-mediated immunity to early antigens }\end{array}$ & $\begin{array}{l}\text { - } \text { reduced TLR expression } \\
\text { - chronic inflammation including macrophage infiltration, increased neutrophils and } \\
\text { lymphocytes in the epidermis } \\
\text { - } \quad \text { impaired NK cell function } \\
\text { - } \quad \text { impaired IFN pathways } \\
\text { - } \quad \text { reduced numbers of antigen presenting cells and impaired antigen presentation } \\
\text { - Th2 response } \\
\text { - } \quad \text { Treg infiltrate } \\
\text { - } \quad \text { few or no CD4 T cells at site } \\
\text { - low CD4:CD8 ratio } \\
\text { - limited cell-mediated immune response } \\
\text { - } \quad \text { increased likelihood of antibodies to capsid }\end{array}$ \\
\hline
\end{tabular}


TLRs 1-3 and 6-9 is significantly increased in cytological samples from HPV infected patients whose disease resolved, whereas expression is reduced from baseline levels in patients with progressive disease [26]. Of the TLRs tested, it was only the expression of TLR4 that did not significantly differ between those with persistent infections and those whose infections resolved. Other studies suggest that HPV may hijack the regulation of TLR pathways. TLR activation in vitro in primary human KCs is regulated by HPV16 E6 and E7 expression. Production of MIP1 $\alpha$ and IL8 is inhibited in E6/E7 expressing $\mathrm{KC}$ stimulated with TLR9 ligand whereas TLR3 and 5 pathways were up-regulated in E6/E7 expressing cells when activated with their respective ligands [23]. In vitro, expression of TLR3, RIG-I and MDA5 are all decreased in KCs expressing genomes from high-risk types [27]. Interestingly, the TLR9 repression did not occur when E6 and E7 from the low-risk genital types were expressed, suggesting that this pathway may enhance only the pathogenesis of high-risk HPV types. The clinical relevance of these studies is yet to be elucidated.

The viral capsid is a potential PAMP. Synthetic HPV16 VLPs made up of L1 alone activate PRR through TLR4 via the MyD88 signaling pathway, initiating T-independent class switch in B cells in the mouse [28]. Human B cells do not express TLR4 [29] and Lenz et al. [30] found no evidence for VLP signaling through TLR1-9 in another "nonprofessional' APC, the plasmacytoid dendritic cell (DC). The immunogenicity of VLP suggests that some form of PRR signalling does occur, however the exact mechanism by which this might occur in HPV infection is yet to be fully elucidated.

NK and NKT cells contribute to the anti-viral innate immune response: NK cells are one of two effector cell types, the other being iNKT cells, of the innate immune response that may contribute to clearance of HPV infection. NK cells constitutively express IFN $\gamma$, granzyme and perforin and so are effectively 'primed' to initiate an anti-viral immune response. Their activation is dependent on the presence of type 1 IFNs and proinflammatory cytokines, such as IL12 and IL18. Following activation, they mediate their cytotoxicity by granzyme and perforin dependent mechanisms. Granzymes reside in the cytoplasm of T cells and NK cells and activate caspases in target cells, triggering apoptosis. Perforin released from the cytoplasmic granules of $\mathrm{T}$ cells and NK cells assembles to make a pore in the target cell, causing membrane damage and lysis.

NK cells detect decreased MHC I expression on cells. The E7 oncoprotein transcriptionally represses TAP1 and LMP2, components of the MHC I processing and presentation pathway and the MHC I heavy chain [31, 32], resulting in reduced MHC I expression on the surface of HPV-infected cells. E7 knock-down with siRNA restores MHC I expression on HPV transformed cells and reduces the cytotoxity of human peripheral blood-derived NK cells against those cells [33]. There are increased numbers of NK cells in SIL, located primarily in the stroma beneath the site of infection and with only a few cells infiltrating the epidermis [34]. On the face of it, the paucity of NK cells in the epidermis in direct contact with infected $\mathrm{KC}$ suggests that NK cytotoxicity, requiring cell-to-cell contact, may not be a significant factor in the regulation of HPV infection.
However NK cells may have other functions that might also contribute to a lack of response to HPV. NK cells can interact with APC and acquire MHC II molecules from them, suppressing CD4 $\mathrm{T}$ cells by presenting antigen without costimulation [35]. Furthermore, a functional impairment of NK cytolysis and cytokine release by NK cells extracted from blood of recurrent respiratory papillomatosis (RRP) patients when cultured with target cells suggests that even when activated, NK cells may be ineffective in their response to HPV [36], although others have reported that NK cells from patients with RRP do not differ from normal [37].

Invariant NKT cells are CD1d-restricted $\mathrm{T}$ cells that express the invariant T cell receptor $\alpha$ chain. NKT cells are not normally found in any significant numbers in normal epidermis but can infiltrate into skin in some pathological conditions [38]. CD1d-deficient mice have defective NKT cells and their functional loss has only modest defects on clearance of viral infection $[39,40]$. Skin resident NKT cells are critical for immune suppression following grafting of K14E7 transgenic mouse skin onto an MHC matched immune competent mouse [41]. NKT cell numbers in HPVinfected lesions have not been studied, however CD1d expression is generally low in infected tissues [42]. The CD1d NKT cell activation signal is down-regulated by HPV E5, which may contribute to viral immune evasion by preventing NKT cell activation [42].

The anti-viral effects of IFN are disrupted by HPV infection: One of the first lines of defense triggered as a consequence of the innate immune detection of viral infection is the type 1 interferon (IFN) response of the infected cell. Type 1 IFNs confer an 'anti-viral' state on the infected cell and its neighbours by initiating the transcription of a number of genes involved in innate and adaptive immune responses, apoptosis and inhibition of virus growth [43]. HPV E6 and E7 genes disrupt the type 1 IFN response by disrupting signal transduction pathways through mechanisms that include the inhibition of STAT phosphorylation, relocalising STATs from the nucleus and by binding and inactivating other pathway members such as IRF-1 (reviewed in [44]). IFNא, a type 1 IFN secreted from $\mathrm{KCs}$, also has anti-viral properties [45]. IFNא expression is epigenetically repressed in cells expressing E6 and E7 from high-risk types [46]. The number of mechanisms evolved by HPV to subvert the IFN response substantiates its importance in protection against viral infection. Furthermore, some efficacy has been shown when IFNo is used therapeutically. The therapeutic introduction of IFN $\alpha$ into patients following excisional treatment of anal condyloma reduced rates of recurrence [47] and is associated with increased likelihood of regression for cervical dysplasia [48].

\section{Regression is Associated with Activation of an Adaptive Immune Response to HPV}

Several studies have shown a clear relationship between $\mathrm{T}$ cell infiltration into the site of a lesion and disease regression, supporting a role for an adaptive $\mathrm{T}$ cell response in lesion regression. In particular, in animal models of regression, infiltration is a common feature associated with its initiation. In the rabbit, ROPV CD11c cells increase in number in the lesion, in early regression, followed by CD4 and $\mathrm{CD} 8+\mathrm{T}$ cells [49]. In the canine oral papillomavirus 
model the predominant infiltrate associated with regression is CD4 and CD8+ T cells [50].

Antigen presentation is the initiating event of the adaptive immune response: Initiation of a primary adaptive immune response is dependent on antigen uptake and presentation by professional APCs, which possess costimulatory molecules that participate in the engagement of naïve $T$ cells to ensure that the $T$ cell is appropriately activated on recognition of peptide and MHC. In the absence of co-stimulation, $\mathrm{T}$ cells become anergic and are then unable to be activated even when exposed to peptide presented on professional APCs. Unlike other epitheliuminfecting viruses such as HSV, which infect APCs in addition to $\mathrm{KC}$, the highly selective tropism of papillomaviruses restricts infection to $\mathrm{KCs}$, which is likely to favor either ignorance or anergy to HPV.

Sampling of the skin microenvironment by the immune system is primarily the responsibility of the skin APCs, which can be divided into the LC in the epidermis, and a selection of DC types in the dermis. LC are the only APCs that infiltrate and reside in the epidermis, and are therefore in proximity to infected $\mathrm{KC}$. However LC are not essential for initiation of a primary immune response. The langerin positive dermal DC present antigen to T cells in the lymph node and this can occur in the absence of LC [8]. It has been reported that both LC and DC can take up HPV VLPs in vitro, however consequential cellular activation (increased expression of co-stimulatory markers and secretion of IL-12) only occurs in DC [51,52]. Uptake of the VLP is immunemediated rather than an active viral infection, and occurs following binding of the VLP on the DC via the FcyRIII receptor [52]. If the lack of APC activation following uptake also extends to HPV infection in vivo, virion uptake by LC would result in an anergic immune response.

Following infection with low or high-risk HPV types (with the exception of $\beta$ types), LC are typically significantly reduced in number at the site of infection as a consequence of viral regulation of the microenvironment [53]. In $\beta$ types, where LC may be present, $\mathrm{T}$ regs are also readily found [53]. E-cadherin expression, which is required for retention of LC in the epidermis, is reduced on infected KCs, limiting adhesion between KC and LC [54]. Expression of MIP3 $\alpha$, a chemokine produced by $\mathrm{KC}$ to signal LC precursors to migrate into the skin, is also reduced in high-risk HPV16 infected KC [55]. The typical loss of LC in HPV infections contrasts with inflammatory HPV lesions (characterized by a cellular infiltrate), where there is an increase in numbers of LC in the epidermis, as well as an influx of dDC [56]. Overall, these data suggest that reduction of LC numbers at the site of infection is important for HPV persistence. Evidence supports the remaining LC in HPV lesions being defective for activation of effector memory $\mathrm{T}$ cells in the skin and instead may contribute to maintenance of Tregs in the lesion.

The involvement of $\mathrm{LC}$ in regression of papillomavirus lesions is unclear. In COPV, there is an infiltration of $\mathrm{CD} 11 \mathrm{c}+$ cells early in disease regression suggesting APC infiltration, however this cell population could not be fully characterized due to the paucity of antibodies available with specificity for rabbit cells [49]. In the canine oral papillomavirus model there were no significant changes in
$\mathrm{CD} 1 \mathrm{a}+\mathrm{LC}$ in regressing lesions [50]. There are clear differences between these models and human cervical HPV infections with high-risk types and the role of LC and dDC, in particular those that are langerin+, remains to be fully elucidated.

CD4+ and CD8+ $T$ cells are likely the major effector cells associated with disease regression: The virus-specific $\mathrm{T}$ cells of the adaptive response consist of CD4+ and CD8+ populations, both of which are associated with regression in the rabbit and dog oral papillomavirus models. CD8+ T cells have traditionally been thought to play a major role in elimination of viral infection, secreting IFN $\gamma$ and displaying cytolytic effects mediated by molecules such as granzyme and perforin. CD4+ T cells also secrete IFN $\gamma$ and instead mediate killing primarily by engagement with ligands for death receptors such as Fas [57] or TRAIL [58], resulting in caspase-mediated apoptosis.

$\mathrm{CD} 4+\mathrm{T}$ cells recognize antigen in the context of MHC II. Sources of antigen include free virus particles or debris from infected cells. Viral antigens taken up by APCs are degraded through the proteasome and incorporated into MHC II on the cell surface. CD8+ T cells respond to antigen presented in the context of MHC I following infection of professional APCs, however there is no evidence to support productive infection of these cells by HPV. Instead it is most likely that cross presentation of HPV peptides on MHC I occurs.

The evidence of a role for $\mathrm{CD} 8+\mathrm{T}$ cell in disease regression is mixed. In a comparison of $\mathrm{CD} 8+\mathrm{T}$ cell responses to E6 and E7 using ELISPOT in individuals with incident infection (new infection following negative PCR tests) or prevalent HPV 16 or 18 infection (lasting $\geq 4$ months), there was no significant difference in the frequency of positivity between patients whose infections cleared (33 $\%$ and those with persistent disease (40\%) [59]. In CIN2/3 lesions, CD8 $+\mathrm{T}$ cells were at a higher frequency in the epidermis of tissues that went on to regress. These cells were characterized as activated, antigen experienced and $\alpha 4 \beta 7+$. Their presence in the epidermis was associated with vascular expression of the ligand for $\alpha 4 \beta 7$, MAdCAM- 1 , which is an adhesion molecule important in T cell homing [60].

$\mathrm{CD} 4+\mathrm{T}$ cells provide 'help', primarily through secretion of IL-2, to ensure that CD8+ T cells are effectively activated to carry out their cytolytic role. CD4+ T cells also directly contribute to viral clearance in the absence of CD8+ T cells. In HSV infection of mice, CD4 T cells are essential for the reduction of the viral burden following intravaginal HSV rechallenge after selective depletion of either CD4+ or CD8+ $\mathrm{T}$ cells [63].

There is evidence of a role for CD4+ T cells in HPV infection. In regressing CIN1 lesions the trend is for increased CD4:CD8 ratios in the stroma [61]. Conversely, there is a general trend for reduced numbers of CD4+ T cells in progressing high-risk HPV lesions with increased grade of CIN $[61,62]$. In the periphery, helper T cell responses to E2 correlate with regression in individuals with LSIL whereas reactivity to E1 and E2 class I restricted epitopes is associated with disease persistence [64]. In normal healthy individuals memory CD4+ T-helper responses against E2 and E6 are detectable, but are impaired in HPV16+ cervical 
cancer patients [65]. Unlike E2 and E6, responses to E7 are poor in healthy individuals [66]. In contrast, in HPV16+ HSIL E7-specific Th1 responses are associated with regression, while the same individuals were unresponsive to an E6 peptide pool [67]. HPV-specific helper T cell responses correlate with regression irrespective of the grade of the lesion. However, the target antigens associated with regression may differ depending on the lesion grade.

Regulatory $T$ cells are associated with persistent disease: Regulatory $\mathrm{T}$ cells (Tregs) are CD4+CD25+FoxP3+ $\mathrm{T}$ lymphocytes that have an immunosuppressive action, which appears to be primarily aimed at preventing the immune response to self-antigen [68]. Tregs function as antiinflammatory $\mathrm{T}$ cells and their secretion of IL-10 may affect the differentiation of DC, inhibiting their cytokine secretion [69]. Selective elimination of Tregs in murine models, particularly when used in combination with other treatments, induces regression of established tumours, implicating Tregs in a suppressive role in the immune response to malignancy.

Tregs have been found in persistent HPV infections. In genital warts, their frequency increases with the size of the lesion [70]. This implicates Tregs in active repression of the immune response to infection with HPV types 6 and 11. Tregs have been found in lesions caused by other HPV types including HPV16 [71]. In lesions caused by $\beta$ types, Tregs were also observed and as is typical for HPV lesions, were located predominantly in the stroma [53].

The presence of Tregs has been associated with expression of the CXCL12 chemokine, which is not expressed in normal skin but is detected in the basal layers of HPV-infected epidermis [72]. CXCL12, also known as stromal cell-derived factor (SDF-1), binds to CXCR4 (CD184) and is strongly chemotactic for lymphocytes and monocytes. The authors propose that its expression in HPV lesions enhances Treg responses. There is evidence of a role for Tregs in HPV16 persistence of infection, with significantly higher frequencies being found in lesions that also contained potentially immune protective IL-2-producing T-helper cells [73].

An antibody response to $\mathrm{HPV}$ is not a requirement for disease regression: There is no doubt that antibodies to the viral coat protein, L1, are extremely effective in preventing disease following immunization with VLPs. However even in the presence of persistent genital HPV infections with common low- and high-risk types, $30-50 \%$ of those infected don't develop antibodies to HPV [74]. Seroconversion is also far from universal after incident infection. In a large prospective cohort study, evidence of seroconversion was seen in around half of women who tested positive for HPV16 or HPV 31 and only around $30 \%$ of those with infected with HPV 18 or HPV45 [75]. Importantly, in women with lowgrade HPV16 lesions a trend towards neutralising antibodies and persistence has been observed, suggesting that the presence of antibodies to L1 from natural infection may be an indicator of poor outcome [76].

\section{CONCLUSIONS}

Persistent infections with HPV occur primarily because the virus can subvert the immune response, allowing its continued presence in an immune competent host. Ultimately this is not sustained as the majority of lesions do eventually regress following a specific, adaptive cell-mediated immune response to the virus.

There is still much to learn about the innate response to HPV. Our understanding of the effects of inflammatory infiltrates that may occur at the time of initial infection on regression of early disease is poor. However chronic inflammation, with sustained inflammatory infiltrates of macrophages and neutrophils is associated with persistence of infection. The virus capsid and viral DNA are potential PAMPs but their immune activating effects in HPV infection are not fully understood. HPV interferes with TLR pathways and repression of TLR expression is related to the persistence of infection. The roles of other PRR and the innate effector cells, NK and NKT cells, are less clear whereas the pathways of disruption of the anti-viral effector molecules, the IFNs, by HPV are well understood.

Activation of APC and antigen presentation in the lymph nodes is necessary for initiation of a primary response to an infecting agent. LC, the APC resident in the epidermis, are typically reduced in persistent HPV lesions from a range of HPV types. In tissues infected with $\beta$ HPV types where LC are present there is also high numbers of Tregs, which are likely to have an immune suppressive effect. Remaining LC in HPV lesions may contribute to regulation of local memory $\mathrm{T}$ cells, rather than having a role in initiation of primary responses to HPV. $\mathrm{dDC}$ are now considered to be the primary immune activating APC. The current understanding of their function in HPV infection is limited and this is an area of research that should be further advanced. For example, it is not clear how HPV antigen is sampled by the $\mathrm{dDC}$, which are not in close proximity to the infected $\mathrm{KC}$.

Regression is associated with a cell-mediated response to the early HPV antigens. CD4 responses to antigens including E2, E6 and E7 have been measured and other early antigens are also likely targets. Regression is associated with infiltration of CD4+ and CD8 $+\mathrm{T}$ cells, whereas CD8+ T cells alone do not appear to be sufficient for regression. In contrast, Tregs infiltrate persisting lesions, contributing to local immunosuppression at the site of infection.

Although there is some understanding of the immune events associated with persistence and regression of HPV infection, a number of questions remain unanswered. Most importantly, the immunological switch that converts persistence to regression is not known. The virus itself is a master-manipulator of the immune response, driving persistence to sustain long-term infection. We need to fully understand the numerous ways that the virus subverts immune function in order to allow us to tip the balance in the favor of the host, rather than the virus. The continuing need for improved diagnostic markers of prognosis and the relatively unrealized potential for effective immunotherapies to treat HPV infection drives the quest for a better understanding of the interactions between the virus and the immune system that lead to regression or allow persistence of HPV infection.

\section{ABBREVIATIONS}

$$
\begin{aligned}
& \mathrm{APC}=\text { Antigen presenting cell } \\
& \mathrm{dDC}=\text { Dermal dendritic cell } \\
& \mathrm{HPV}=\text { Human papillomavirus }
\end{aligned}
$$




$$
\begin{aligned}
& \mathrm{HSV}=\text { Herpes simplex virus } \\
& \text { IFN }=\text { Interferon } \\
& \mathrm{KC}=\text { Keratinocyte } \\
& \mathrm{LC}=\text { Langerhans cell } \\
& \text { LSIL }=\text { Low-grade squamous intraepithelial lesion } \\
& \text { MHC }=\text { Major histocompatibility complex } \\
& \text { NLR }=\text { Nucleotide oligomerisation domain-like receptor } \\
& \text { PAMP }=\text { Pathogen-associated molecular pattern } \\
& \text { PRR }=\text { Pattern recognition receptor } \\
& \text { RLR }=\text { RIL-1-like receptor } \\
& \text { RRP }=\text { Recurrent respiratory papillomatosis } \\
& \text { SDF-1 = Stromal cell-derived factor } \\
& \text { TLR }=\text { Toll-like receptor } \\
& \text { Treg = Regulatory T cell }
\end{aligned}
$$

\section{CONFLICT OF INTEREST}

The author confirms that this article content has no conflicts of interest.

\section{ACKNOWLEDGEMENTS}

This research was supported through funding from the Health Research Council of New Zealand.

\section{REFERENCES}

[1] Ho GY, Bierman R, Beardsley L, Chang CJ, Burk RD. Natural history of cervicovaginal papillomavirus infection in young women. $\mathrm{N}$ Engl $\mathrm{J}$ Med 1998; 338: 423-8.

[2] Maran A, Amella CA, Di Lorenzo TP, Auborn KJ, Taichman LB, Steinberg BM. Human papillomavirus type 11 transcripts are present at low abundance in latently infected respiratory tissues. Virology 1995; 212: 285-94.

[3] Maglennon GA, McIntosh P, Doorbar J. Persistence of viral DNA in the epithelial basal layer suggests a model for papillomavirus latency following immune regression. Virology 2011; 414: 153-63.

[4] Black AP, Ardern-Jones MR, Kasprowicz V, et al. Human keratinocyte induction of rapid effector function in antigen-specific memory CD4+ and CD8+ T cells. Eur J Immunol 2007; 37: 1485-93.

[5] Foster CA, Yokozeki H, Rappersberger K, et al. Human epidermal T cells predominantly belong to the lineage expressing alpha/beta $\mathrm{T}$ cell receptor. J Exp Med 1990; 171: 997-1013.

[6] Bursch LS, Wang L, Igyarto B, et al. Identification of a novel population of Langerin+ dendritic cells. J Exp Med 2007; 204: 3147-56.

[7] van de Ven R, van den Hout MF, Lindenberg JJ, et al. Characterization of four conventional dendritic cell subsets in human skin-draining lymph nodes in relation to T-cell activation. Blood 2011; 118: 2502-10.

[8] Henri S, Poulin LF, Tamoutounour S, et al. CD207+ CD103+ dermal dendritic cells cross-present keratinocyte-derived antigens irrespective of the presence of Langerhans cells. J Exp Med 2010; 207: 189-206.

[9] Bedoui S, Whitney PG, Waithman J, et al. Cross-presentation of viral and self antigens by skin-derived CD103+ dendritic cells. Nat Immunol 2009; 10: 488-95.

[10] Poppe WA, Drijkoningen M, Ide PS, Lauweryns JM, Van Assche FA. Lymphocytes and dendritic cells in the normal uterine cervix. An immunohistochemical study. Eur J Obstet Gynecol Reprod Biol 1998; 81:277-82.

[11] Clark RA, Chong B, Mirchandani N, et al. The vast majority of CLA+ T cells are resident in normal skin. J Immunol 2006; 176: 4431-9.

[12] Boyman O, Conrad C, Tonel G, Gilliet M, Nestle FO. The pathogenic role of tissue-resident immune cells in psoriasis. Trends Immunol 2007; 28: 51-7.

[13] Wakim LM, Waithman J, van Rooijen N, Heath WR, Carbone FR. Dendritic cell-induced memory $\mathrm{T}$ cell activation in nonlymphoid tissues. Science 2008; 319: 198-202.
[14] Jiang X, Clark RA, Liu L, Wagers AJ, Fuhlbrigge RC, Kupper TS. Skin infection generates non-migratory memory $\mathrm{CD} 8+\mathrm{T}(\mathrm{RM})$ cells providing global skin immunity. Nature 2012; 483: 227-31.

[15] Woodland DL, Kohlmeier JE. Migration, maintenance and recall of memory T cells in peripheral tissues. Nat Rev Immunol 2009; 9:153-61.

[16] Giraldo PC, de Carvalho JB, do Amaral RL, d et al. Identification of immune cells by flow cytometry in vaginal lavages from women with vulvovaginitis and normal microflora. Am J Reprod Immunol 2012; 67: 198-205.

[17] Doorbar J. Molecular biology of human papillomavirus infection and cervical cancer. Clin Sci (Lond) 2006; 110: 525-41.

[18] Agaiby AD, Dyson M. Immuno-inflammatory cell dynamics during cutaneous wound healing. J Anat 1999; 195( Pt 4): 531-42.

[19] Hagari Y, Budgeon LR, Pickel MD, Kreider JW. Association of tumor necrosis factor-alpha gene expression and apoptotic cell death with regression of Shope papillomas. J Invest Dermatol 1995; 104: 526-9.

[20] Hammes LS, Tekmal RR, Naud P, imran.yassen Macrophages, inflammation and risk of cervical intraepithelial neoplasia (CIN) progression--clinicopathological correlation. Gynecol Oncol 2007; 105: $157-65$.

[21] Castle PE, Hillier SL, Rabe LK, et al. An association of cervical inflammation with high-grade cervical neoplasia in women infected with oncogenic human papillomavirus (HPV). Cancer Epidemiol Biomarkers Prev 2001; 10: 1021-7.

[22] Kovacic MB, Katki HA, Kreimer AR, Sherman ME. Epidemiologic analysis of histologic cervical inflammation: relationship to human papillomavirus infections. Hum Pathol 2008; 39: 1088-95.

[23] Hasan UA, Bates E, Takeshita F, et al. TLR9 expression and function is abolished by the cervical cancer-associated human papillomavirus type 16. J Immunol 2007; 178: 3186-97.

[24] Lebre MC, van der Aar AM, van Baarsen L, et al. Human keratinocytes express functional Toll-like receptor 3, 4, 5, and 9. J Invest Dermatol 2007; 127:331-41.

[25] Feldmeyer L, Keller M, Niklaus G, Hohl D, Werner S, Beer HD. The inflammasome mediates UVB-induced activation and secretion of interleukin-1beta by keratinocytes. Curr Biol 2007; 17: 1140-5.

[26] Daud, II, Scott ME, Ma Y, Shiboski S, Farhat S, Moscicki AB Association between toll-like receptor expression and human papillomavirus type 16 persistence. Int J Cancer 2011; 128: 879-86.

[27] Reiser J, Hurst J, Voges M, et al. High-risk human papillomaviruses repress constitutive kappa interferon transcription via E6 to prevent pathogen recognition receptor and antiviral-gene expression. J Virol 2011; 85: 11372-80.

[28] Yang R, Murillo FM, Delannoy MJ, et al. B lymphocyte activation by human papillomavirus-like particles directly induces Ig class switch recombination via TLR4-MyD88. J Immunol 2005; 174: 7912-9.

[29] He B, Qiao X, Cerutti A. CpG DNA induces IgG class switch DNA recombination by activating human $\mathrm{B}$ cells through an innate pathway that requires TLR9 and cooperates with IL-10. J Immunol 2004; 173: 4479-91.

[30] Lenz P, Lowy DR, Schiller JT. Papillomavirus virus-like particles induce cytokines characteristic of innate immune responses in plasmacytoid dendritic cells. Eur J Immunol 2005; 35: 1548-56.

[31] Li H, Ou X, Xiong J, Wang T. HPV16E7 mediates HADC chromatin repression and downregulation of MHC class I genes in HPV16 tumorigenic cells through interaction with an MHC class I promoter Biochem Biophys Res Commun 2006; 349: 1315-21.

[32] Georgopoulos NT, Proffitt JL, Blair GE. Transcriptional regulation of the major histocompatibility complex (MHC) class I heavy chain, TAP1 and LMP2 genes by the human papillomavirus (HPV) type 6b, 16 and 18 E7 oncoproteins. Oncogene 2000; 19: 4930-5.

[33] Bottley G, Watherston OG, Hiew YL, Norrild B, Cook GP, Blair GE. High-risk human papillomavirus E7 expression reduces cell-surface MHC class I molecules and increases susceptibility to natural killer cells. Oncogene 2008; 27: 1794-9.

[34] Renoux VM, Bisig B, Langers I, et al. Human papillomavirus entry into NK cells requires CD16 expression and triggers cytotoxic activity and cytokine secretion. Eur J Immunol 2011; 41: 3240-52.

[35] Nakayama M, Takeda K, Kawano M, Takai T, Ishii N, Ogasawara K. Natural killer (NK)-dendritic cell interactions generate MHC class IIdressed NK cells that regulate CD4+ T cells. Proc Natl Acad Sci USA 2011; 108: 18360-5.

[36] Bonagura VR, Hatam LJ, Rosenthal DW, et al. Recurrent respiratory papillomatosis: a complex defect in immune responsiveness to human papillomavirus-6 and -11. APMIS 2010; 118: 455-70. 
[37] Naiman HB, Doyle AT, Ruben RJ, Kadish AS. Natural cytotoxicity and interferon production in patients with recurrent respiratory papillomatosis. Ann Otol Rhinol Laryngol 1984; 93: 483-7.

[38] Gober MD, Fishelevich R, Zhao Y, Unutmaz D, Gaspari AA. Human natural killer $\mathrm{T}$ cells infiltrate into the skin at elicitation sites of allergic contact dermatitis. J Invest Dermatol 2008; 128: 1460-9.

[39] Ilyinskii PO, Wang R, Balk SP, Exley MA. CD1d mediates T-celldependent resistance to secondary infection with encephalomyocarditis virus $(\mathrm{EMCV})$ in vitro and immune response to $\mathrm{EMCV}$ infection in vivo. J Virol 2006; 80: 7146-58.

[40] Huber S, Sartini D, Exley M. Role of CD1d in coxsackievirus B3induced myocarditis. J Immunol 2003; 170: 3147-53.

[41] Mattarollo SR, Rahimpour A, Choyce A, Godfrey DI, Leggatt GR, Frazer IH. Invariant NKT cells in hyperplastic skin induce a local immune suppressive environment by IFN-gamma production. J Immunol 2010; 184:1242-50.

[42] Miura S, Kawana K, Schust DJ, et al. CD1d, a sentinel molecule bridging innate and adaptive immunity, is downregulated by the human papillomavirus (HPV) E5 protein: a possible mechanism for immune evasion by HPV. J Virol 2010; 84: 11614-23.

[43] Perry AK, Chen G, Zheng D, Tang H, Cheng G. The host type I interferon response to viral and bacterial infections. Cell Res 2005; 15: $407-22$.

[44] Kanodia S, Fahey LM, Kast WM. Mechanisms used by human papillomaviruses to escape the host immune response. Curr Cancer Drug Targets 2007; 7: 79-89.

[45] LaFleur DW, Nardelli B, Tsareva T, et al. Interferon-kappa, a novel type I interferon expressed in human keratinocytes. J Biol Chem 2001; 276: 39765-71.

[46] Rincon-Orozco B, Halec G, Rosenberger S, et al. Epigenetic silencing of interferon-kappa in human papillomavirus type 16-positive cells. Cancer Res 2009; 69: 8718-25.

[47] Fleshner P, Freilich M. Adjuvant interferon for anal condyloma. Dis Colon Rectum 1994; 37: 1255-9.

[48] Palomba M, Melis GB. Oral use of interferon therapy in cervical human papillomavirus infection. Clin Ter 2000; 151: 59-61.

[49] Wilgenburg BJ, Budgeon LR, Lang CM, Griffith JW, Christensen ND. Characterization of immune responses during regression of rabbit oral papillomavirus infections. Comp Med 2005; 55: 431-9.

[50] Nicholls PK, Moore PF, Anderson DM, et al. Regression of canine oral papillomas is associated with infiltration of $\mathrm{CD} 4+$ and CD8+ lymphocytes. Virology 2001; 283: 31-9.

[51] Fausch SC, Da Silva DM, Rudolf MP, Kast WM. Human papillomavirus virus-like particles do not activate Langerhans cells: a possible immune escape mechanism used by human papillomaviruses. J Immunol 2002; 169: 3242-9.

[52] Da Silva DM, Fausch SC, Verbeek JS, Kast WM. Uptake of human papillomavirus virus-like particles by dendritic cells is mediated by Fcgamma receptors and contributes to acquisition of $\mathrm{T}$ cell immunity. $\mathrm{J}$ Immunol 2007; 178: 7587-97.

[53] Leong CM, Doorbar J, Nindl I, Yoon HS, Hibma MH. Loss of epidermal Langerhans cells occurs in human papillomavirus alpha, gamma, and mu but not beta genus infections. J Invest Dermatol 2010; 130: $472-80$.

[54] Matthews K, Leong CM, Baxter L, et al. Depletion of Langerhans cells in human papillomavirus type 16-infected skin is associated with E6mediated down regulation of E-cadherin. J Virol 2003; 77: 8378-85.

[55] Guess JC, McCance DJ. Decreased migration of Langerhans precursorlike cells in response to human keratinocytes expressing human papillomavirus type $16 \mathrm{E} 6 / \mathrm{E} 7$ is related to reduced macrophage inflammatory protein-3alpha production. J Virol 2005; 79: 14852-62.

[56] Nakayama Y, Asagoe K, Yamauchi A, et al. Dendritic cell subsets and immunological milieu in inflammatory human papilloma virus-related skin lesions. J Dermatol Sci 2011; 63: 173-83.
[57] Lowin B, Hahne M, Mattmann C, Tschopp J. Cytolytic T-cell cytotoxicity is mediated through perforin and Fas lytic pathways. Nature 1994; 370: 650-2.

[58] Kayagaki N, Yamaguchi N, Nakayama M, et al. Involvement of TNFrelated apoptosis-inducing ligand in human $\mathrm{CD} 4+\mathrm{T}$ cell-mediated cytotoxicity. J Immunol 1999; 162: 2639-47.

[59] Coleman HN, Moscicki AB, Farhat S, Gupta S, Wang X, Nakagawa M. CD8 T-cell responses in incident and prevalent human papillomavirus types 16 and 18 infections. ISRN Obstet Gynecol 2012; 2012: 854237.

[60] Trimble CL, Clark RA, Thoburn C, et al. Human papillomavirus 16associated cervical intraepithelial neoplasia in humans excludes CD8 T cells from dysplastic epithelium. J Immunol 2010; 185: 7107-14.

[61] Monnier-Benoit S, Mauny F, Riethmuller D, et al. Immunohistochemical analysis of CD4+ and CD8+ T-cell subsets in high risk human papillomavirus-associated pre-malignant and malignant lesions of the uterine cervix. Gynecol Oncol 2006; 102: 2231.

[62] Bell MC, Edwards RP, Partridge EE, et al. CD8+ T lymphocytes are recruited to neoplastic cervix. J Clin Immunol 1995; 15: 130-6.

[63] Milligan GN, Bernstein DI, Bourne N. T lymphocytes are required for protection of the vaginal mucosae and sensory ganglia of immune mice against reinfection with herpes simplex virus type 2. J Immunol 1998; 160: 6093-100.

[64] Dillon S, Sasagawa T, Crawford A, et al. Resolution of cervical dysplasia is associated with T-cell proliferative responses to human papillomavirus type 16 E2. J Gen Virol 2007; 88: 803-13.

[65] de Jong A, van Poelgeest MI, van der Hulst JM, et al. Human papillomavirus type 16-positive cervical cancer is associated with impaired CD4+ T-cell immunity against early antigens E2 and E6. Cancer Res 2004; 64: 5449-55.

[66] Welters MJ, de Jong A, van den Eeden SJ, et al. Frequent display of human papillomavirus type 16 E6-specific memory t-Helper cells in the healthy population as witness of previous viral encounter. Cancer Res 2003; 63: 636-41.

[67] Peng S, Trimble C, Wu L, et al. HLA-DQB1*02-restricted HPV-16 E7 peptide-specific $\mathrm{CD} 4+\mathrm{T}$-cell immune responses correlate with regression of HPV-16-associated high-grade squamous intraepithelial lesions. Clin Cancer Res 2007; 13: 2479-87.

[68] Loddenkemper C, Hoffmann C, Stanke J, et al. Regulatory (FOXP3+) $\mathrm{T}$ cells as target for immune therapy of cervical intraepithelial neoplasia and cervical cancer. Cancer Sci 2009; 100: 1112-7.

[69] Kitching AR, Holdsworth SR. The emergence of TH17 cells as effectors of renal injury. J Am Soc Nephrol 2011; 22: 235-8.

[70] Cao Y, Zhao J, Lei Z, et al. Local accumulation of FOXP3+ regulatory $T$ cells: evidence for an immune evasion mechanism in patients with large condylomata acuminata. J Immunol 2008; 180: 7681-6.

[71] Scott ME, Ma Y, Kuzmich L, Moscicki AB. Diminished IFN-gamma and IL-10 and elevated Foxp3 mRNA expression in the cervix are associated with CIN 2 or 3. Int J Cancer 2009; 124: 1379-83.

[72] Jaafar F, Righi E, Lindstrom V, et al. Correlation of CXCL12 expression and FoxP3+ cell infiltration with human papillomavirus infection and clinicopathological progression of cervical cancer. Am J Pathol 2009; 175: 1525-35.

[73] Molling JW, de Gruijl TD, Glim J, et al. CD4(+)CD25hi regulatory Tcell frequency correlates with persistence of human papillomavirus type 16 and $\mathrm{T}$ helper cell responses in patients with cervical intraepithelial neoplasia. Int J Cancer 2007; 121: 1749-55.

[74] Carter JJ, Koutsky LA, Hughes JP, et al. Comparison of human papillomavirus types 16,18 , and 6 capsid antibody responses following incident infection. J Infect Dis 2000; 181: 1911-9.

[75] Wang SS, Schiffman M, Shields TS, et al. Seroprevalence of human papillomavirus-16, $-18,-31$, and -45 in a population-based cohort of 10000 women in Costa Rica. Br J Cancer 2003; 89: 1248-54.

[76] Ochi $\mathrm{H}$, Matsumoto $\mathrm{K}$, Kondo $\mathrm{K}$, et al. Do neutralizing antibody responses generated by human papillomavirus infections favor a better outcome of low-grade cervical lesions? J Med Virol 2012; 84: 1128-34. 